\title{
1 An agent-based model of adaptation of holobionts with different microbial 2 symbiont transmission modes
}

3 Running title: Simulation of holobiont adaptation to stress

4 Siao Ye ${ }^{1 * *}$, Zhu Liu $^{2^{*}}$ and Evan Siemann ${ }^{1}$

$5 \quad{ }^{\mathbf{1}}$ Department of Biosciences, Rice University, Houston, Texas, 77005, USA

$6 \quad{ }^{2}$ Department of Preventive Medicine, Keck School of Medicine, University of Southern

7 California, Los Angeles, 90007, USA

$8{ }^{\#}$ Corresponding author: Department of Biosciences, Rice University, 6100 S. Main St., Houston,

$9 \quad$ Texas, 77005, USA; E-mail: thomasyesiao@gmail.com

10 *Siao Ye and Zhu Liu contributed equally to this work. Author order was determined both

11 alphabetically and in order of increasing seniority.

\section{KEYWORDS}

13 Endosymbiosis, holobiont, agent-based modeling, adaptation, stress 


\section{ABSTRACT}

16 The hologenome theory suggests that holobionts (host plus symbiont) with hosts that are only

17 able to adapt slowly may be able to persist in deteriorating environmental conditions via rapid

18 adaptation of their microbial symbionts. The effectiveness of such symbiont adaptation may vary

19 depending on whether symbionts are passed directly to offspring (vertical transmission) or

20 acquired from the environment (horizontal transmission). However, it has been suggested that

21 holobionts with horizontal transmission cannot pass down their symbionts faithfully, preventing

22 adaptation at the holobiont level because of host-symbiont disassociation between generations.

23 Here we used an agent-based model to investigate whether holobionts with horizontal microbial

24 symbiont transmission can adapt to increasing stress solely through symbiont adaptation and

25 compared their adaptation to holobionts with vertical transmission. We found that holobionts

26 with either transmission mode were able to adapt to increasing abiotic stress solely via symbiont

27 adaptation. Moreover, those with horizontal transmission were more competitive than those with

28 vertical transmission when hosts were able to selectively associate with the most suitable

29 symbionts. However, those with horizontal transmission were less competitive than those with

30 vertical transmission when symbiont establishment was random. Our results support the

31 hologenome theory and demonstrate that holobionts with horizontal microbial symbiont

32 transmission could adapt to increasing abiotic stress via their symbionts. We also showed that

33 whether holobionts with horizontal or vertical symbiont transmission are favored in increasingly

34 stressful conditions depends on the ability of hosts to recognize and foster microbial symbionts

35 that confer stress tolerance. 


\section{IMPORTANCE}

37 Symbiotic organisms such as reef building corals are sensitive to environmental perturbations

38 due to anthropogenic disturbances or climate change, and it is critical to understand whether they

39 are able to adapt to previously unfavorable conditions. To date, studies have focused on the

40 impacts of existing microbial symbiont variation on holobiont stress tolerance but here we use

41 agent-based models to explore holobiont adaptation via symbiont adaptation. We studied both

42 deterministic and stochastic processes in holobiont adaptation by investigating the following four

43 factors: holobiont transmission modes, a host's ability to recognize tolerance-conferring

44 symbionts, a symbiont's mutational variance, and rate of stress increase. Our simulation provides

45 a comprehensive understanding of holobiont adaptation under stress, which not only has

46 implications for future endangered symbiotic species management, but also provides fresh

47 insight into species evolution as proposed by the hologenome theory. 


\section{INTRODUCTION}

49 Research on symbiotic organisms has suggested that symbiont variation can drive holobiont

50 (host plus symbiont) phenotypic diversification, and influence holobiont fitness (1). In addition

51 to host genetic changes, symbiont genetic changes can alter the holobiont phenotype and

52 therefore impact holobiont adaptation (2). Zilber-Rosenberg and Rosenberg proposed that rapid

53 holobiont adaptation could take place even when host adaptation is slow, because symbiont

54 adaptation and composition shifts could be rapid (3). A diversity of potential symbiont partners

55 are rich resources for holobionts, which might be critical for holobionts to adapt to increasing

56 environmental stress such as global warming $(4,5)$.

57 Compared to hosts, symbionts have large population sizes and short generation times, and

58 may adapt to ambient changes (6), yet it is unclear whether symbiont adaptation alone could

59 drive holobiont adaptation. Most studies on holobionts focused on variation in symbiont

60 composition rather than symbiont adaptation. For example, researchers found cnidarians

61 associating with different symbiont strains differ in their thermal tolerances $(5,7)$, and thermal

62 tolerance of aphids can be controlled by their symbiotic bacteria $(8,9)$. Such changes in

63 holobiont phenotype are mainly based on existing symbiont diversity and can be observed within

64 one generation. However, experiments testing holobiont adaptation via symbiont adaptation and

65 composition shifts would be difficult because they require tracking of symbiont composition as

66 well as holobiont phenotypes over a long time and so is less explored. Chakravarti et al. (10)

67 pointed out that assisted evolution in symbionts can be applied to improve holobiont stress

68 tolerance, and they were able to detect its impact on symbionts stress tolerance, but not on the

69 holobiont stress tolerance. Some field observations also suggest holobiont adaptation can be

70 achieved through symbiont adaptation. Rodriguez et al. (11) discovered the stress tolerance 
71 conferred by a symbiotic fungi to host plants is habitat dependent. Another study on corals also

72 revealed local adaptation existed in symbionts and leads to holobiont divergence in their thermal

73 tolerance, in which the symbiotic algae collected from warmer reefs maintained higher resistance

74 to heat compared to those from cooler habitats even after multiple asexual generations (12).

75 Nevertheless, it is hard to monitor symbiont adaptation in the field over evolutionary time scales

76 and track its impact on holobiont fitness. A simulation model would be appropriate to study

77 holobiont adaptation via symbiont adaptation $(13,14)$, which is lacking in holobiont research.

To investigate whether holobionts can adapt to stressful environments via symbiont

79 adaptation, it is critical to understand whether holobionts with either horizontal symbiont

80 transmission (" $\mathrm{H}$ " hereafter) or vertical symbiont transmission ("V" hereafter) are both able to

81 pass their adaptive symbionts to offspring, and which type is better at coping with stress. Some

82 researchers argue that passing down adaptive symbionts to offspring is unstable for holobionts

83 conducting horizontal transmission, which makes the hologenome theory less plausible $(15,16)$.

84 They suggest the symbiont community could be shaped simply by opportunity and

85 environmental filters, and the transmission fidelity is low $(15,16)$. In addition, opposite opinions

86 are given when discussing H's and V's adaptability. In vertical transmission, individuals inherit

87 symbionts directly from parents, which is similar to classical gene inheritance, so adaptive

88 symbionts from parents can be passed to offspring (17). But there are drawbacks associated with

89 this transmission mode; symbionts picked up by offspring might only be a subset of their

90 parents', so symbiont diversity could decrease dramatically through evolutionary time, and

91 become maladaptive once the ambient conditions change $(18,19)$. On the other hand, individuals

92 conducting horizontal transmission pick up a variety of symbionts in the neighborhood, so they

93 might respond to environmental changes faster by associating with stress tolerant symbionts (4, 
94 20). However, the disassociation of symbionts and hosts between generations could lead to loss

95 of adaptive symbionts and colonization of virulent symbionts $(21,22)$. Most studies on microbe

96 transmission and adaptation in the past investigated how different transmission modes affect

97 virulence $(17,22-24)$, and explored the dynamics of virulence-transmission trade off instead of

98 considering them as a selection unit (25-27), because their systems emphasize antagonistic

99 rather than mutualistic relations between the host and the symbiont. Roughgarden (28) conducted

100 a pioneering simulation of holobiont evolution and found both vertically transmitted and

101 horizontally transmitted agents were able to evolve as a holobiont unit. But she used the number

102 of microbes to determine holobiont fitness in her model, and did not study the interactions

103 between the environment and holobionts. Because of the complex interactions among the host,

104 the symbiont, and the environment, combined with the different symbiont acquisition and

105 retention mechanisms in $\mathrm{Hs}$ and Vs, it is hard to predict whether Hs and Vs are able to adapt to

106 climate change, and which mode is more adaptive with increasing environmental stress.

107 Holobionts with different transmission modes could differ in their novel symbiont acquisition

108 and retention, which makes it hard to predict whether they are able to adapt to climate change,

109 and which modes is more adaptive.

Here we use agent-based modeling to explore how holobionts with different transmission

111 modes respond to increasing stress because such models are flexible and able to handle complex

112 problems $(29,30)$. Interestingly, such topics are better explored in cultural transmission, where

113 researchers explore how knowledge information (e.g., language, knowledge, etc.) is transmitted

114 between generations under different environment regimes (31). In cultural transmission,

115 vertically inherited information (from parents) is more conservative than obliquely acquired

116 (from elder people excluding parents or ancestors) (32), which resembles vertically transmitted 
117 symbionts and horizontally transmitted symbionts. Some studies reveal oblique transmission is

118 favored when the environment fluctuates, and is necessary in driving language evolution, while

119 vertical transmission is preferred when the environment is stable $(33,34)$. However, there could

120 be fundamental differences between cultural transmission and symbiont transmission. For

121 example, there is no increasing stress such as global warming in cultural transmission, so these

122 studies do not provide insights into whether both transmission modes allow holobiont adaption to

123 directional selection. In addition to transmission modes, we considered three other important

124 factors that might impact holobiont persistence in changing climate: 1) the host's ability to select

125 adaptive symbionts, 2) the rate at which stress increases, and 3) symbiont mutational variance.

126 We propose that the ability to select adaptive symbionts is a key factor when studying

127 holobiont adaptation, even though it is barely discussed, if at all, in previous research. Because

128 the amount of resources and space an organism can provide to symbionts is limited, holobiont

129 fitness is largely determined by the relative amount of adaptive symbionts within it $(3,35)$. So if

130 symbiont acquisition is completely random, then Hs will have no advantages over Vs even if Hs

131 have access to a larger symbiont pool from which they can choose partners, because the chances

132 of getting adaptive and maladaptive symbionts are the same. In addition, drift might have larger

133 impacts on Vs because the bottleneck effect is stronger in them due to the smaller effective

134 symbiont population size (18). On the contrary, if symbiont acquisition is not random but can be

135 determined by the host, then Hs might be more likely to associate with adaptive symbionts than

136 Vs, and drift impacts would be limited. Such symbiont selection by hosts is not uncommon and

137 has be found in both Hs and Vs, for example, solitary wasps block transmission of nonnative

138 symbionts to offspring, and sorghum selectively increases monoderm bacteria during drought to 
139 improve plant growth (36-38). Thus we tested the impacts of drift on Hs and Vs adaptation by

140 switching on and off the ability of holobionts to select for adaptive symbionts in our model.

141 Whether holobionts are able to adapt to the changing environment via symbiont

142 adaptation also depends on how fast stress increases and how fast symbionts adapt. Holobionts

143 such as corals are assumed to rely on the symbiont rather than the host to adapt to rising sea

144 temperature, because corals are living close to their thermal limits and the temperature is

145 increasing quickly $(39,40)$. Compared to the host, the symbionts are abundant with short

146 generations, so they are more likely the key to holobiont adaptation $(3,41)$. Because most

147 symbionts (e.g., bacteria, algae, fungi) reproduce asexually $(10,42)$, we consider mutation the

148 major source of generating novel traits. Incorporation of mutation in our model makes the traits

149 continuous and dynamic instead of static and discrete, and generates intra-individual as well as

150 inter-individual symbiont variation. Such variation is necessary in understanding the complex

151 interactions between holobionts and the environment, and interactions among holobionts (43, 44).

152 Because population trait variance is sensitive to the magnitude of mutation (i.e., mutational

153 variance) rather than mutation rate, it is important to know how mutational variance might affect

154 distribution of symbiont traits and thus holobiont adaptation $(45,46)$. Large mutational variance

155 may have greater impacts on horizontally transmitted holobionts, because once an extreme

156 symbiont strain arises, it can be transmitted throughout the population and may be hard to lose,

157 while it will be constrained in certain lineages in vertically transmitted holobionts. But large

158 mutational variance could also produce lineages that have accumulated extreme symbionts in Vs

159 when drift is strong. By varying the speed at which stress increases and mutational variance,

160 combined with controlling drift, we explored how the selection-mutation-drift balance interacted

161 with transmission modes. 
Our goal was to present a model to test whether holobiont adaptation can be driven by

163 associated symbiont changes, which involves symbiont mutation, acquisition, amplification and

164 transmission. The agent-based model we used simulated changes at the environment level, the

165 holobiont level, and the symbiont level, by varying four main parameters: transmission mode, the

166 ability to select adaptive symbionts, the rate of environmental change and the magnitude of

167 symbiont mutation. It involves interactions between the environment and the holobionts, among

168 the holobionts, and between the holobionts and the symbionts. We believe our model could

169 produce novel insights into holobiont adaptation.

\section{RESULTS}

\section{Test case}

172 We verified that when agents were selectively neutral in our basic model, the fixation was

173 random at a 1:1 $\mathrm{V}$ to $\mathrm{H}$ initial ratio (Fig. 1).

\section{Increasing temperature}

175 Regardless of whether transmission is random or optimal, both Vs and Hs were able to adapt to

176 increasing temperature when the rate of temperature increase was low and mutation variance was

177 high (Fig. 2).

Generally, holobionts persisted in high mutation variance and low temperature rate of

179 change conditions, but went extinct in low mutation variance and high temperature rate of

180 change conditions.

However, the transmission mode did affect the relative fitness of Hs and Vs (Fig. 3).

182 When transmission was completely random, Vs had higher fitness (Wilcoxon test, $\mathrm{p}=0.0002$ ) and

183 longer time to extinction (Wilcoxon test, $\mathrm{p}=0.03$ ) compared to Hs for the same parameter 
184 combination, and their gene values also had higher correlation with the temperature (Wilcoxon

185 test, $\mathrm{p}=0.0017)$. When transmission was optimal, Hs had higher fitness and longer time to

186 extinction, as well as higher correlation between gene values and temperature.

When both agent types were present at the same time, we were able to compare the ratio

188 of Hs and Vs (Fig. 4), which tells their relative competitiveness. In both random transmission

189 and optimal transmission models, Hs and Vs became extinct in large step/small mutation_sd

190 regions, and coexisted in small step/small mutation_sd regions. In the rest of regions, Vs

191 persisted in the random transmission model (Fig. 4a), and Hs persisted in the optimal

192 transmission model (Fig. 4b).

We also checked how virulence would affect the relative competitiveness of Hs and Vs

194 by reducing birthrate of Hs by $5 \%$ at mutation_sd $=0.001$ and step=0 in both models. This

195 resulted in Vs fixation in both models.

To explore how drift shapes symbiont distribution in the random transmission model, we

197 compared fitness variance between individuals and within individuals (Fig. 5). We found Vs had

198 larger variance among individuals (Wilcoxon test, $\mathrm{p}<0.001$ ), but less variance within individuals

199 (Wilcoxon test, $\mathrm{p}<0.001$ ). This means symbiont distributions in each vertical holobiont were

200 more homogenous than in each horizontal holobiont, but vertical holobionts were more

201 heterogeneous than horizontal holobionts across the holobiont fitness landscape.

\section{DISCUSSION}

We present here an agent-based model that investigates the adaptation ability of vertically

204 transmitted holobionts and horizontally transmitted holobionts to increasing stress through

205 symbiont adaptation. By introducing intraspecific trait variation and enabling evolution, we were 
able to study how selection-mutation-drift affected the population dynamics of holobionts with

207 different transmission modes. Our model clearly demonstrates that both Vs and Hs are able to

208 adapt to increasing environmental stress via symbiont adaptation, and their relative

209 competitiveness depends on whether symbiont transmission is random or not. As long as the

210 stress does not increase at a speed that will drive the holobionts to extinction rapidly, symbiont

211 adaptation alone could increase holobiont stress tolerance.

Our model suggests the answer to a long debated question: can horizontally transmitted

213 species be considered as a selection unit even though the symbiont disassociates from the host

214 during holobiont life history $(16,47)$ ? We showed that although offspring do not necessarily

215 inherit symbionts from their parents directly, they could still acquire adapted symbionts. We

216 found stress tolerance conferred by symbionts improved over time as the mean symbiont gene

217 value increased along with increasing temperature. This was because holobionts whose symbiont

218 values better matched the temperature were more likely to survive, and they passed their adapted

219 symbionts to individuals in the next generation. In other words, because selection acts on the

220 population level and drives the symbiont to confer higher stress tolerance, individual holobionts

221 sampled from a symbiont pool that was adapting, and thus became adapted. This process looks

222 like a Lamarckian evolution because agents acquire stress tolerance by associating with stress-

223 resistant symbionts horizontally (48), but it is actually Darwinian evolution on the whole

224 symbiont population level since the symbiont gene frequency changes due to selection against

225 maladapted holobionts. Osmanovic et al. (49) found that selection on toxin tolerance of

226 vertically transmitted bacteria alleviated holobiont stress in a long-lasting toxic environment.

227 They suggested that horizontally transmitted symbionts can also be selected for and will confer

228 higher stress tolerance to hosts over time which is similar to our results here. Acquiring adaptive 
traits via associating with horizontally transmitted symbionts is commonly observed in nature. In

230 aphids, the secondary symbionts are considered to form a horizontal gene pool which facilitate

231 aphid adaptation (50). Redman et al. discovered fungal-free plants that received Curvularia

232 isolates (a type of fungal endophyte) from geothermal regions exhibited improved thermal

233 tolerance, which also suggests stress tolerance could be acquired horizontally (51). In addition,

234 research on corals with horizontally transmitted symbionts revealed surprisingly high fidelity in

235 symbionts across generations, indicating unknown mechanisms that maintain symbiont stability

236 in Hs (52). Thus we propose that symbiont-mediated holobiont adaptation is plausible in both Hs

237 and Vs.

240 competitiveness. Researchers have opposite opinions regarding which transmission mode is

241 more advantageous because of following reasons: on one hand, Hs could exploit a broader range

242 of symbionts, and are likely to take up adaptive symbionts from the environment $(21,53)$, while

243 Vs can only inherit parents' symbionts, which may be maladaptive once the environment

244 changes $(54,55)$. On the other hand, adaptive symbionts could be passed down faithfully in

245 vertical transmission, but get lost during horizontal transmission $(4,15)$. Our results suggest both

246 arguments are partially correct, but are case dependent because symbiont acquisition could be

247 random or non-random, which has significant impacts on the outcome (56). When transmission

248 is random, Hs have no advantage even though the holobionts have access to a wider range of

249 symbionts, because the chances of taking up adaptive and maladaptive symbionts are equal; but

250 when they are able to recognize adaptive symbionts, they can pick up the fittest symbionts from

251 a larger pool compared to the limited choices Vs have, which greatly improves their performance. 
In random transmission scenarios, drift is stronger in Vs and can drive symbiont fixation

253 in offspring lineages rapidly because they experience strong bottleneck effects given limited

254 symbiont options in parents $(18,57)$; in contrast, Hs offspring tend to maintain their parents'

255 optimum according to the law of large numbers, because they sample from a larger symbiont

256 pool (58). Our simulation of Hs and Vs without selection has shown that symbiont similarity is

257 higher within each $\mathrm{V}$ than within each $\mathrm{H}$ (i.e., within host symbiont variation is larger in $\mathrm{H}$

258 individuals than in V individuals), but lower among Vs than among Hs (i.e., between-host

259 symbiont variation is lower among Hs than among Vs). This is consistent with studies on corals,

260 that found symbionts among vertically transmitted corals are more differentiated, and symbionts

261 among horizontally transmitted corals are less differentiated $(52,59)$. In psyllids, some hosts are

262 found to harbor only clonal symbiont lineages due to their vertical transmission mode, and the

263 low diversity within the holobiont suggests a strong bottleneck effect (60). Given enough time,

264 vertically transmitted symbionts could become so specific in host lineages that they will have

265 reduced genome sizes and cannot escape from hosts (61). The strategies of Vs and Hs are thus

266 different when facing environmental changes. Vs produce lineages that are distinctive in their

267 stress tolerance, while Hs produce individuals more likely to persist in all conditions. Because

268 maladaptive lineages that are dominated by maladaptive symbionts can be removed quickly from

269 V populations, while maladaptive symbionts can persist in H populations through coexisting

270 with adaptive symbionts, Vs are able to respond to environmental changes faster than Hs, and

271 thus adapt faster when symbiont transmission is random.

273 population, while they are constrained in certain lineages in the $\mathrm{V}$ population, because of the

274 larger potential host population available for each symbiont in Hs (21). From this perspective, 
275 having maladaptive symbionts is similar to having parasites, which may provide additional

276 explanation for why higher virulence is often associated with horizontal transmission $(24,27)$.

277 Because maladaptive symbionts can be acquired by holobionts other than direct descendants,

278 they could persist in Hs just like infectious diseases, and decrease Hs' overall fitness. In contrast,

279 V individuals have a smaller sampling pool, which is determined by their parent, and offspring

280 that are quickly dominated by maladaptive strains due to drift can be selected against (57), so

281 overall fitness improves faster under selection. In short, once maladaptive symbionts arise in Hs,

282 they can be taken up and spread through the population and are unlikely to be eliminated, but in

283 Vs they can be trapped within lineages and be selected against, causing lower overall fitness in

284 Hs than Vs. In an extreme scenario when the stress increasing speed is zero (i.e., under a

285 constant environment), although both populations could persist for the whole simulation, Vs had

286 slightly higher fitness especially when the mutational variance is large, where maladaptive

287 symbionts are likely to arise and spread in Hs. Given enough time, Vs should be able to

288 outcompete Hs (Fig. 5a). This resembles results of a study on virulence in pathogens, where

289 vertical transmitted pathogens were selected for to prevent host from acquiring more virulent

290 pathogens horizontally (27), and is consistent with study results of cultural transmission that

291 suggest Vs are favored under stable conditions $(33,62)$. Our simulation also support the

292 hypothesis that vertically transmitted holobionts such as corals may be more robust to climate

293 change (63), but conditional on them conducting random transmission.

295 opposite result that Hs were better at tracking a changing environment. Such "recognition" of

296 suitable symbionts is not well explored in previous studies $(28,34)$, yet their impacts on the

297 outcome was very important in our simulation. We think such optimal transmission is equivalent 
to the positive transgenerational feedback proposed by Xue and Leibler (64). They suggested phenotypes of parents are reinforced in offspring, so organisms could adapt to varying environments. The positive transgenerational effect has been observed in some empirical studies, which is probably caused by epigenetic processes $(65,66)$. Our previous experiment on green hydra also showed that positive transgenerational effects could occur in holobionts simply via

303 transmission of acclimated symbionts. The model presented here is slightly different from that 304 described in Xue and Leibler's and examples mentioned above, because here we are focusing on 305 symbiont evolution rather than host evolution, and the phenotypes are distributed continuously

306 rather than discretely. Nevertheless, the acquired symbionts were derived from those hosted by

307 holobionts in the previous generations, which were more likely to be decedents of adaptive 308 symbionts than maladaptive symbionts in their ancestors. Because the temperature in our model 309 changes in one direction, the fittest new mutants are more likely to arise from the dominant, 310 fittest strain in parent, instead of from a less fit strain. Such horizontal transmission of stress311 tolerant symbionts has been observed in corals, which suggests holobiont adaptation can be 312 achieved through transmission of adaptive symbionts $(67,68)$. By having access to symbionts

313 released by holobionts other than their direct ancestors, Hs were able to selectively associate

314 with the fittest strain that arose in the population which is in contrast to Vs. The ability to pick up 315 the fittest symbionts, which is similar to copying from the most successful individuals in social 316 learning, increases Hs fitness and allows them to adapt faster than Vs (69).

318 symbionts: the first is via partner choice; the second is via passive up take. Mounting evidence 319 of partner choice is being shown for holobionts (70), for example, plants allocate more resources 
321 cheaters and maximize their fitness during symbiosis. Bobtail squid are able to prevent

322 colonization of deficient luminescent bacteria Vibrio fischeri in their light organ, which could be

323 another case of partner choice (72). Massive coral bleaching could also be a case of hosts

324 ejecting maladaptive symbionts and establishing symbiosis with adaptive ones (40). Corals are

325 able to shift their symbiotic algae from sensitive strains to stress tolerant strains, so they can

326 become more robust to stress such as high temperature and UV (53, 73-75). Thus even coral

327 hosts that have long generation times and may not be able to adapt to warming temperatures

328 quickly enough still have a chance to withstand climate change (3). An interesting example of

329 wasps also shows that parents rather than the offspring may be able to choose symbionts to be

330 transmitted (37), as such transmission could be controlled on a cellular level (76). In addition,

331 even if the host is not able to identify the appropriate partner, they could form symbiosis with

332 symbionts that pass an environment filter. Because symbionts vary in their stress tolerance, a

333 strain that exhibits the highest fitness under certain conditions could outcompete other strains,

334 and then form symbiosis with hosts (77). Hosts taking up these adaptive symbionts would

335 acquire appropriate stress tolerance towards their environment (12). In insects, thermal stress

336 could easily wipe out their thermal sensitive symbiotic bacteria, which could reduce their

337 fecundity $(8,78)$. However, there are strains that could withstand such stress, and introducing

338 these strains into hosts can restore hosts' reproduction and enhance their thermal tolerance $(8$,

339 79). Symbionts that diverge in their niches are able to confer different traits and form association

340 with hosts in distinctive conditions, which facilitates holobiont diversification $(9,50,80)$. These

341 examples provide solid evidence of optimal transmission of symbionts and suggest we should

342 take optimal symbiont transmission into consideration when studying holobiont persistence in

343 stressful environments. 
Regardless of transmission mode and the ability to associate with optimal symbionts or

345 not, holobionts were more likely to persist under stress when their mutational variance was large

346 and stress increasing speed was low. It is not surprising that holobionts were able to persist

347 longer at low rates of increasing temperature, because other studies also suggest rapid increase in

348 stress could quickly wipe out the organisms (45, 81). However, Ayllón et al. (46) did not detect

349 significant impacts of mutational variance on adaptation, and they suggested it was because their

350 simulation ran for a limited number of generations (barely over 100 generations). In contrast, our

351 simulations lasted for 4000 generations. Moreover, whether holobionts went extinct or not

352 seemed to be confined by non-linear combinations of the mutational variance and temperature

353 increasing speed, which requires larger increase in mutational variance at high rates of

354 temperature increase than that at low rates of increase (Fig. 4).

Our models are the first exploring holobiont adaptation to stress, and demonstrate that

356 both Hs and Vs are able to adapt, even when symbionts and hosts are disassociated between

357 generations in Hs. Yet the model could be further expanded by incorporating more factors that

358 might affect the dynamics between $\mathrm{Hs}$ and Vs in different scenarios. For instance, conducting

359 horizontal transmission could be virulent, which might reduce Hs' overall fitness and their

360 competitiveness (27). In jellyfish, horizontal transmission favored symbionts shift to being

361 parasitic, which reduced the growth rate of jellyfish up to 50\% (22). We have applied this only to

362 scenarios when there is no change in temperature, and found a 5\% decrease in Hs' growth rate

363 could greatly reduce their relative frequency in both random transmission and optimal

364 transmission. This implies that under constant environments, vertical transmission with less

365 virulent symbionts would be favored $(17,33,62,82)$. But if we adjust the virulence parameter in

366 temperature increasing scenarios, we will be able to explore how virulence could alter holobiont 
367 population dynamics. Another factor worth exploring could be the tradeoff between thermal

368 tolerance and fitness cost. Studies on corals reveal that symbionts that confer higher thermal

369 tolerance may slow down the growth of corals $(83,84)$. Imposing such constraints may enhance

370 the authenticity of the model because holobionts will not be able to increase their thermal

371 tolerance infinitely. We can also investigate into how Hs and Vs react in other scenarios such as

372 random environments or fluctuating environments. We hope our models could provide a basic

373 framework for holobiont evolution and shed light on symbionts' roles from both ecological and

374 evolutionary perspectives.

\section{MATERIALS AND METHODS}

376 Our model was coded in python 3.0 with the agent-based modeling package "Mesa". Here we

377 present a model description following the ODD (Overview, Design Concepts, Details) protocol

378 by giving a summary of the overall model structure and processes (29). Detailed variables and

379 functions definitions can be found in Appendix A.

380 Purpose

The purpose of the model is to understand whether holobionts with vertical transmission

382 or horizontal transmission could adapt to changing environments solely depending on their

383 symbionts, and how drift and mutation would affect this process. The stress in this model is

384 called "temperature", which is a hypothetical stress without a real world metric because it could

385 also be called "salinity" or "UV intensity". Similarly, each timestep represents one generation

386 time, and has no corresponding real-world time length, because symbiotic species vary in their

387 generation time. To minimize the model complexity, holobionts (the agents) in our model are

388 autotrophic or heterotrophic with unlimited food and so do not need to compete for food, but 
they have to compete for space, which is common in ecosystems such as coral reefs or for plants

$390(41,85)$. Once a grid cell is occupied, it cannot be taken over by another agent unless the

391 previous occupier is dead.

\section{Scales and variables}

The model simulates adaptation of Vs and Hs under different temperature regimes, which

394 contains three hierarchical levels: 1) environment, 2) holobiont, 3) symbiont. The object of the

395 environment level is temperature. The temperature is a standardized hypothetical parameter

396 without units because we are not studying a specific species and a specific stress tolerance, but

397 rather to verify if holobionts can evolve as a unit. Each time step represents one generation, and

398 agents could die or give birth to one offspring. The objects of the second level are the two types

399 of holobionts, Vs and Hs, which only differ in their transmission modes. To track the adaptability

400 of Vs and Hs, agents reproduce asexually and do not switch their transmission modes across

401 generations. This allowed us to better track symbiont compositions of the two agent types over

402 time, and compare fitness of Vs and Hs. The object of the third level, symbiont, is the only factor

403 that determines holobionts' fitness. Because our goal is to test whether both Vs and Hs can adapt

404 via symbiont adaptation, and many holobionts' stress tolerance is largely determined by

405 symbiont stress tolerance (40,86-88), we assume no host impacts on holobiont's stress tolerance

406 in this model. Each agent contains a fixed number of symbionts, and each symbiont is assigned

407 a randomly generated gene value from which the host fitness will be calculated.

408 Scheduling

The model is executed in following steps: 
Initially 2000 agents are generated and randomly distributed on a 50*50 size grid. In

412 cases where both Hs and Vs are presented, each agent has 50\% chance to be $\mathrm{H}$ and $50 \%$ chance

413 to be $\mathrm{V}$. Each agent contains 50 symbionts, whose value is drawn from a normal distribution

414 with mean equal to 0.5 and variance equal to mutational variance, truncated at two standard

415 deviations $(46,89)$. Initial temperature is set at 0.5 as well.

416 (2). Holobiont reproduction

417 At every time step, each agent has a chance to reproduce based on its fitness, which is the

418 mean of the fitness score calculated for its symbionts, assuming all the symbionts within the host

419 will affect the holobiont's fitness. We used a symmetric beta distribution to calculate the fitness

420 score (90), which reaches the maximum when the symbiont's gene value matches current

421 temperature (Fig.6), and reaches a minimum when the mismatch is equal or greater than one, in

422 other words, the current temperature could be either too hot or too cold for the symbiont. A

423 random number is generated and compared to the holobiont's fitness value to decide whether it

424 will reproduce or not. Because conducting horizontal transmission might suppress holobiont

425 reproduction, we introduce a cost parameter "l" that could reduce H's reproduction probability

426 (82).

427 (3). Symbiont transmission

In the default model, drift is introduced by enabling random symbiont transmission in $\mathrm{Hs}$

429 and Vs. V offspring sample symbionts from their parents randomly with replacement, so their

430 symbionts could be a subset of those in their parents. H offspring sample symbionts from other

431 agents around them once they are produced and dispersed (17). This transmission process is 
432 equivalent to picking up symbionts from a common pool constitute of symbionts released from

433 agents (28).

In the optimal transmission model, drift is suppressed by enabling agents to pick the

435 fittest symbionts from parents. As in cases where certain symbiont strains is dominant while the

436 rest strains are marginal, here offspring choose the fittest strain from the parent (in V) or from

437 neighboring agents (in $\mathrm{H}$ ) to make up the symbiont population, and then randomly sample with

438 replacement as in the default model to fill the rest of its symbiont population.

439 (4). Symbiont mutation

440 During holobiont reproduction, the symbiont may mutate by chance once it is acquired by

441 the offspring. The mutation probability of each symbiont strain within the holobiont is set at 0.01

442 (91), given that symbiont generation time is shorter than holobiont generation time while the

443 abundance is large $(92,93)$. The mutated symbiont gene value is drawn from a normal

444 distribution with mean centered on the original symbiont gene value, variance controlled by the

445 mutational variance, truncated at two standard deviation from the mean (46). Since we are

446 interested in whether symbiont gene changes could drive holobiont adaptation, we assume

447 heritability equals one and there is no environmental variance in our model.

448 (5). Holobiont dispersal

449 We use a stepwise function to control the position of a newborn holobiont. Because the

450 further away from the parent the less likely the offspring would occur (94), we set equally high

451 probability within certain distance for the new holobiont to occupy the gird, and equally low

452 probability beyond certain distance for the holobiont to occur. 


\section{3 (6). Selection}

Agent fitness is calculated as described above, except for the decision whether the agent

455 will die or not.

Detailed parameters are documented in appendix A, and each parameter combination was

457 run for 4000 time steps and at least 20 iterations.

\section{Design Concepts}

Emergence: Population dynamics emerge from the reproduction, selection and

460 competition of the two types of agents, embedded with symbiont drift and mutation. Adaptation

461 is driven by interactions between each agent and the environment. optimal transmission model, agents are able to pick the fittest symbionts from their ancestors.

466 which depict the relation between fitness and temperature stress.

472 individuals and the environment. 
474 size over time, relative ratio of the two agent types, mean holobiont optimal temperature, and

475 time to extinction, etc. (95).

\section{Simulation scenarios}

\section{7 (1) Test case}

To test and validate the model, we constructed a basic model that both agent types have

479 equal fixed reproduction and survival probability, so they are selectively neutral and their fitness

480 does not depend on the environment. This could be viewed as two genotypes in a finite

481 population, whose fixation should be random. According to Kimura \& Ohta (57), when the

482 initial relative ratio is 0.5 , either $\mathrm{H}$ or $\mathrm{V}$ will fix at $50 \%$ chance, and if the initial relative ratio

483 moves away from 0.5 , one or the other will be more likely to fix within dramatically less time.

484 (2) Increasing temperature

The temperature was projected to increase at different speed infinitely, which means

486 holobionts would only be able to persist by having novel mutations conferring thermal tolerance

487 higher than that of existing symbionts. This model tested whether both Vs and Hs could keep up 488 with increasing stress, and explored how symbiont mutational variance might affect this process 489 given different increasing speed. For both random transmission model and optimal transmission 490 model, Hs and Vs were simulated in coexistence and in separation.

\section{$491 \quad$ Statistical analysis}

We used paired Wilcoxon rank tests to compare the fitness between Hs and Vs across all

493 the parameter combinations. We summed up the mean holobiont fitness at each time step of 
494 every iteration for a given parameter combination and performed a log transformation. This

495 provides us information that includes both the fitness at each time step as well as the extinction

496 time. We used the same test for Hs and Vs extinction time, correlation between holobiont gene

497 values and the temperature, fitness score variance within holobionts, and fitness score variance

498 between holobionts. 


\section{REFERENCES}

501 1. Hurst GDD. 2017. Extended genomes: Symbiosis and evolution. Interface Focus

$502 \quad 7: 20170001$.

503 2. Haag KL. 2018. Holobionts and their hologenomes: Evolution with mixed modes of

$504 \quad$ inheritance. Genet Mol Biol 41:189-197.

505 3. Zilber-Rosenberg I, Rosenberg E. 2008. Role of microorganisms in the evolution of

506 animals and plants: the hologenome theory of evolution. FEMS Microbiol Rev 32:723-

$507 \quad 735$.

508 4. Byler KA, Carmi-Veal M, Fine M, Goulet TL. 2013. Multiple symbiont acquisition

509 strategies as an adaptive mechanism in the coral Stylophora pistillata. PLoS One 8:e59596.

510 5. Berkelmans R, van Oppen MJ. 2006. The role of zooxanthellae in the thermal tolerance of 511 corals: a 'nugget of hope' for coral reefs in an era of climate change. Proc R Soc B Biol $512 \quad$ Sci 273:2305-2312.

513 6. Lynch M, Gabriel W, Wood AM. 1991. Adaptive and demographic responses of plankton 514 populations to environmental change. Limnol Oceanogr 36:1301-1312.

515 7. Ye S, Bhattacharjee M, Siemann E. 2019. Thermal tolerance in green hydra: Identifying 516 the roles of algal endosymbionts and hosts in a freshwater holobiont under stress. Microb $517 \quad$ Ecol 77:537-545.

518 8. Dunbar HE, Wilson ACC, Ferguson NR, Moran NA. 2007. Aphid thermal tolerance is 519 governed by a point mutation in bacterial symbionts. PLoS Biol 5:e96. 
520 9. Heyworth ER, Ferrari J. 2015. A facultative endosymbiont in aphids can provide diverse

521 ecological benefits. J Evol Biol 28:1753-1760.

522 10. Chakravarti LJ, Beltran VH, van Oppen MJH. 2017. Rapid thermal adaptation in

523 photosymbionts of reef-building corals. Glob Chang Biol 23:4675-4688.

524 11. Rodriguez RJ, Henson J, Van Volkenburgh E, Hoy M, Wright L, Beckwith F, Kim Y-O,

525 Redman RS. 2008. Stress tolerance in plants via habitat-adapted symbiosis. ISME J

$526 \quad 2: 404-416$.

527 12. Howells EJ, Beltran VH, Larsen NW, Bay LK, Willis BL, van Oppen MJH. 2012. Coral

528 thermal tolerance shaped by local adaptation of photosymbionts. Nat Clim Chang 2:116-

$529 \quad 120$.

530 13. Evans MR, Grimm V, Johst K, Knuuttila T, de Langhe R, Lessells CM, Merz M,

531 O’Malley MA, Orzack SH, Weisberg M, Wilkinson DJ, Wolkenhauer O, Benton TG.

532 2013. Do simple models lead to generality in ecology? Trends Ecol Evol 28:578-583.

533 14. Peck SL. 2004. Simulation as experiment: a philosophical reassessment for biological

534 modeling. Trends Ecol Evol 19:530-534.

535 15. Moran NA, Sloan DB. 2015. The hologenome concept: Helpful or hollow? PLOS Biol

$536 \quad 13: \mathrm{e} 1002311$.

537 16. Douglas AE, Werren JH. 2016. Holes in the hologenome: Why host-microbe symbioses $538 \quad$ are not holobionts. MBio 7:e02099.

539 17. Drown DM, Zee PC, Brandvain Y, Wade MJ. 2013. Evolution of transmission mode in $540 \quad$ obligate symbionts. Evol Ecol Res 15:43-59. 
541 18. Mira A, Moran NA. 2002. Estimating population size and transmission bottlenecks in

542 maternally transmitted endosymbiotic bacteria. Microb Ecol 44:137-143.

543 19. Dusi E, Krenek S, Schrallhammer M, Sachse R, Rauch G, Kaltz O, Berendonk TU. 2014.

$544 \quad$ Vertically transmitted symbiont reduces host fitness along temperature gradient. J Evol

$545 \quad$ Biol 27:796-800.

546 20. Bright M, Bulgheresi S. 2010. A complex journey: transmission of microbial symbionts.

$547 \quad$ Nat Rev Microbiol 8:218-230.

548 21. Douglas AE. 1998. Host benefit and the evolution of specialization in symbiosis. Heredity

$549 \quad$ 81: 599-603.

550 22. Sachs JL, Wilcox TP. 2006. A shift to parasitism in the jellyfish symbiont Symbiodinium 551 microadriaticum. Proceedings Biol Sci 273:425-9.

552 23. de Roode JC, Yates AJ, Altizer S. 2008. Virulence-transmission trade-offs and population 553 divergence in virulence in a naturally occurring butterfly parasite. Proc Natl Acad Sci U S $554 \quad$ A $105: 7489-94$.

555 24. Stewart AD, Logsdon JM, Kelley SE. 2005. An empirical study of the evolution of 556 virulence under both horizontal and vertical transmission. Evolution 59:730-9.

557 25. Ferdy J-B, Godelle B. 2005. Diversification of transmission modes and the evolution of $558 \quad$ mutualism. Am Nat 166:613-627.

559 26. Blanquart F, Grabowski MK, Herbeck J, Nalugoda F, Serwadda D, Eller MA, Robb ML, 560 Gray R, Kigozi G, Laeyendecker O, Lythgoe KA, Nakigozi G, Quinn TC, Reynolds SJ, 561 Wawer MJ, Fraser C. 2016. A transmission-virulence evolutionary trade-off explains 
attenuation of HIV-1 in uganda. Elife 5:e20492.

563 27. Lipsitch M, Siller S, Nowak MA. 1996. The evolution of virulence in pathogens with vertical and horizontal transmission. Evolution 50:1729.

28. Roughgarden J. 2018. Holobiont evolution: Model with lineal vs. collective hologenome inheritance. bioRxiv 465310.

29. Grimm V, Berger U, Bastiansen F, Eliassen S, Ginot V, Giske J, Goss-Custard J, Grand T, individual-based and agent-based models. Ecol Modell 198:115-126.

572 30. Patt A, Siebenhüner B. 2005. Agent based modeling and adaptation to climate change.

$573 \quad$ Vierteljahrshefte zur Wirtschaftsforsch 74:310-320.

574 31. Fogarty L, Feldman MW. 2015. Cultural evolution: Theory and models, p. 401-408. In

576 32. Gong T. 2010. Exploring the roles of horizontal, vertical, and oblique transmissions in $577 \quad$ language evolution. Adapt Behav 18:356-376.

578 33. McElreath R, Strimling P. 2008. When natural selection favors imitation of parents. Curr $579 \quad$ Anthropol 49:307-316.

580 34. Ram Y, Liberman U, Feldman MW. 2019. Vertical and oblique cultural transmission 581 fluctuating in time and in space. Theor Popul Biol 125:11-19. 
582 35. Itoh H, Jang S, Takeshita K, Ohbayashi T, Ohnishi N, Meng XY, Mitani Y, Kikuchi Y.

583 2019. Host-symbiont specificity determined by microbe-microbe competition in an insect

584 gut. Proc Natl Acad Sci U S A 116:22673-22682.

585 36. Xu L, Naylor D, Dong Z, Simmons T, Pierroz G, Hixson KK, Kim YM, Zink EM,

586 Engbrecht KM, Wang Y, Gao C, DeGraaf S, Madera MA, Sievert JA, Hollingsworth J,

587 Birdseye D, Scheller H V., Hutmacher R, Dahlberg J, Jansson C, Taylor JW, Lemaux PG,

588 Coleman-Derr D. 2018. Drought delays development of the sorghum root microbiome and

589 enriches for monoderm bacteria. Proc Natl Acad Sci U S A 115:E4284-E4293.

590 37. Kaltenpoth M, Roeser-Mueller K, Koehler S, Peterson A, Nechitaylo TY, Stubblefield JW,

591 Herzner G, Seger J, Strohm E. 2014. Partner choice and fidelity stabilize coevolution in a

592 Cretaceous-age defensive symbiosis. Proc Natl Acad Sci U S A 111:6359-64.

593 38. Werner GDA, Kiers ET. 2015. Partner selection in the mycorrhizal mutualism. New Phytol 205: 1437-1442.

39. Lough JM, Anderson KD, Hughes TP. 2018. Increasing thermal stress for tropical coral reefs: 1871-2017. Sci Rep. Nature Publishing Group 8:1-8.

597 40. Berkelmans R, Van Oppen MJH. 2006. The role of zooxanthellae in the thermal tolerance of corals: A "nugget of hope" for coral reefs in an era of climate change. Proc R Soc B Biol Sci 273:2305-2312.

600 41. Allemand D, Furla P. 2018. How does an animal behave like a plant? Physiological and molecular adaptations of zooxanthellae and their hosts to symbiosis. C R Biol 341:276280. 
603 42. Faeth SH. 2009. Asexual fungal symbionts alter reproductive allocation and herbivory over time in their native perennial grass hosts. Am Nat 173:554-565.

43. Bolnick DI, Amarasekare P, Araújo MS, Bürger R, Levine JM, Novak M, Rudolf VHW, Schreiber SJ, Urban MC, Vasseur DA. 2011. Why intraspecific trait variation matters in community ecology. Trends Ecol Evol 26:183-92.

44. Courbaud B, Vieilledent G, Kunstler G. 2012. Intra-specific variability and the competition-colonisation trade-off: Coexistence, abundance and stability patterns. Theor Ecol 5:61-71.

45. Burger R, Lynch M. 1995. Evolution and extinction in a changing environment: A quantitative-genetic analysis. Evolution 49:151. environmental change. Ecol Modell 326:36-53.

47. Theis KR, Dheilly NM, Klassen JL, Brucker RM, Baines JF, Bosch TCG, Cryan JF, contains Lamarckian aspects within a Darwinian framework. Environ Microbiol 11:29592962. 
bacteria supports emergence of Lamarckian-like adaptation of the system as a whole. Biol

50. Henry LM, Peccoud J, Simon J-C, Hadfield JD, Maiden MJC, Ferrari J, Godfray HCJ. Biol 23:1713-1717.

629 51. Redman RS, Sheehan KB, Stout RG, Rodriguez RJ, Henson JM. 2002. Thermotolerance generated by plant / fungal symbiosis. Science 298:1581.

631 52. Quigley KM, Willis BL, Bay LK. 2017. Heritability of the Symbiodinium community in vertically- and horizontally-transmitting broadcast spawning corals. Sci Rep 7:8219.

53. Buddemeier RW, Baker AC, Fautin DG, Jacobs JR. 2004. The adaptive hypothesis of bleaching, p. 427-444. In Rosenberg, PE, Loya, PY (eds.), Coral Health and Disease. Springer Berlin Heidelberg. Holobionts as units of selection and a model of their population dynamics and evolution. symbiont acquisition in obligate mutualisms. Curr Biol 27:3711-3716.e3.

641 56. Grube, M, White, JF, Seckbach, J (eds.). 2010. Symbioses and Stress. Springer, 642 Dordrecht.

643 57. Kimura M, Ohta T. 1969. The average number of generations until fixation of a mutant 644 gene in a finite population. Genetics 61:763-71. 
645 58. Dekking M. 2005. A modern introduction to probability and statistics : understanding why

646 and how. Springer.

647 59. Loh WKW, Loi T, Carter D, Hoegh-Guldberg O. 2001. Genetic variability of the symbiotic dinoflagellates from the wide ranging coral species Seriatopora hystrix and Acropora longicyathus in the Indo-West Pacific. Mar Ecol Prog Ser 222:97-107.

60. Morrow JL, Hall AAG, Riegler M. 2017. Symbionts in waiting: the dynamics of incipient endosymbiont complementation and replacement in minimal bacterial communities of psyllids. Microbiome 5:58.

61. Fisher RM, Henry LM, Cornwallis CK, Kiers ET, West SA. 2017. The evolution of hostsymbiont dependence. Nat Commun 8:15973. under fluctuating selection. Proc Natl Acad Sci U S A 115:E1174-E1183.

63. Swain TD, Westneat MW, Backman V, Marcelino LA. 2018. Phylogenetic analysis of symbiont transmission mechanisms reveal evolutionary patterns in thermotolerance and host specificity that enhance bleaching resistance among vertically transmitted Symbiodinium. Eur J Phycol 53:443-459.

64. Xue B, Leibler S. 2016. Evolutionary learning of adaptation to varying environments through a transgenerational feedback. Proc Natl Acad Sci U S A 113:11266-11271. environments. Non-Genetic Inherit 1:38-50. 
climate change experiments: Where do we go from here? Glob Chang Biol 24:13-34.

667 67. Quigley KM, Willis BL, Kenkel CD. 2019. Transgenerational inheritance of shuffled symbiont communities in the coral Montipora digitata. Sci Rep 9:13328.

669 68. Cumbo V, van Oppen M, Baird A. 2018. Temperature and Symbiodinium physiology

670 affect the establishment and development of symbiosis in corals. Mar Ecol Prog Ser

$671 \quad 587: 117-127$.

672 69. Rendell L, Boyd R, Cownden D, Enquist M, Eriksson K, Feldman MW, Fogarty L,

673 Ghirlanda S, Lillicrap T, Laland KN. 2010. Why copy others? Insights from the social 674 learning strategies tournament. Science 328:208-13.

675 70. Shapiro JW, Turner PE. 2014. The impact of transmission mode on the evolution of 676 benefits provided by microbial symbionts. Ecol Evol 4:3350-3361.

677 71. Denison RF. 2000. Legume sanctions and the evolution of symbiotic cooperation by $678 \quad$ rhizobia. Am Nat 156:567-576.

679 72. Visick KL, Foster J, Doino J, McFall-Ngai M, Ruby EG. 2000. Vibrio fischeri lux genes 680 play an important role in colonization and development of the host light organ. J Bacteriol $681 \quad 182: 4578-86$

682 73. Wietheger A. 2012. Coral bleaching resistance vs susceptibility: the role of antioxidant 683 activity in symbiotic dinoflagellates. Victoria University of Wellington. PhD theis.

684 74. Brown BE. 1997. Coral bleaching: Causes and consequences. Coral Reefs 16:S129-S138.

685 75. Jones AM, Berkelmans R, Oppen MJH van, Mieog JC, Sinclair W. 2008. A community 
change in the algal endosymbionts of a scleractinian coral following a natural bleaching

76. Koga R, Meng X-Y, Tsuchida T, Fukatsu T. 2012. Cellular mechanism for selective vertical transmission of an obligate insect symbiont at the bacteriocyte-embryo interface. Proc Natl Acad Sci U S A 109:E1230-7.

691 77. Karim W, Nakaema S, Hidaka M, Karim W, Nakaema S, Hidaka M. 2015. Temperature effects on the growth rates and photosynthetic activities of Symbiodinium cells. J Mar Sci Eng 3:368-381.

78. Wernegreen JJ. 2012. Mutualism meltdown in insects: Bacteria constrain thermal adaptation. Curr Opin Microbiol 15: 255-262.

79. Montllor CB, Maxmen A, Purcell AH. 2002. Facultative bacterial endosymbionts benefit pea aphids Acyrthosiphon pisum under heat stress. Ecol Entomol 27:189-195.

80. Richardson SL. 2001. Endosymbiont change as a key innovation in the adaptive radiation of Soritida (foraminifera). Paleobiology 27:262-289.

81. Matuszewski S, Hermisson J, Kopp M. 2015. Catch me if you can: Adaptation from standing genetic variation to a moving phenotypic optimum. Genetics 200:1255-1274.

82. Antonovics J, Wilson AJ, Forbes MR, Hauffe HC, Kallio ER, Leggett HC, Longdon B, Okamura B, Sait SM, Webster JP. 2017. The evolution of transmission mode. Philos Trans R Soc B Biol Sci. The Royal Society 372: 20160083. 
12.

84. Jones A, Berkelmans R. 2010. Potential costs of acclimatization to a warmer climate:

85. Selosse M-A, Baudoin E, Vandenkoornhuyse P. 2004. Symbiotic microorganisms, a key

for ecological success and protection of plants. C R Biol 327:639-648.

712 86. Giauque H, Connor EW, Hawkes C V. 2019. Endophyte traits relevant to stress tolerance,

713 resource use and habitat of origin predict effects on host plants. New Phytol 221:2239-

2249.

715 87. Tchernov D, Gorbunov MY, de Vargas C, Narayan Yadav S, Milligan AJ, Häggblom M,

716 Falkowski PG. 2004. Membrane lipids of symbiotic algae are diagnostic of sensitivity to

717 thermal bleaching in corals. Proc Natl Acad Sci U S A 101:13531-5.

718 88. Rodriguez R, Redman R. 2008. More than 400 million years of evolution and some plants

719 still can't make it on their own: Plant stress tolerance via fungal symbiosis. Journal of

$720 \quad$ Experimental Botany 59:1109-1114.

721 89. Baskett ML, Gaines SD, Nisbet RM. 2009. Symbiont diversity may help coral reefs survive moderate climate change. Ecol Appl 19:3-17.

723 90. Streck NA. 2004. A temperature response function for modeling leaf growth and

724 development of the African violet (Saintpaulia ionantha Wendl.). Ciência Rural 34:55-62.

725 91. Clark JR, Daines SJ, Lenton TM, Watson AJ, Williams HTP. 2011. Individual-based

726 modelling of adaptation in marine microbial populations using genetically defined

727 physiological parameters. Ecol Modell 222:3823-3837. 
728 92. Császár NBM, Ralph PJ, Frankham R, Berkelmans R, van Oppen MJH. 2010. Estimating

729 the potential for adaptation of corals to climate warming. PLoS One 5:e9751.

730 93. Dillon MM, Sung W, Lynch M, Cooper VS. 2018. Periodic variation of mutation rates in

731 bacterial genomes associated with replication timing. MBio 9:e01371-18.

732 94. Portnoy S, Willson MF. 1993. Seed dispersal curves: behavior of the tail of the

733 distribution. Evolutionary Ecology 7:25-44.

734 95. Grimm V, Wissel C. 2004. The intrinsic mean time to extinction: a unifying approach to 735 analysing persistence and viability of populations. Oikos 105:501-511.

736

\section{ACKNOWLEDGEMENTS}

738 We thank Dr. Rudy Guerra and Dr. Marek Kimmel for providing assistance to our model

739 development. 




743 Figure 1: Fixation of holobiont transmission types under selectively neutral conditions and

744 equal starting abundances of vertical and horizontal transmission types. Blue lines indicate

745 the proportion of holobionts that have vertical transmission (V) and red lines indicate the

746 proportion that have horizontal transmission $(\mathrm{H})$. Each simulation is represented by a

747 single blue line and single red line. 

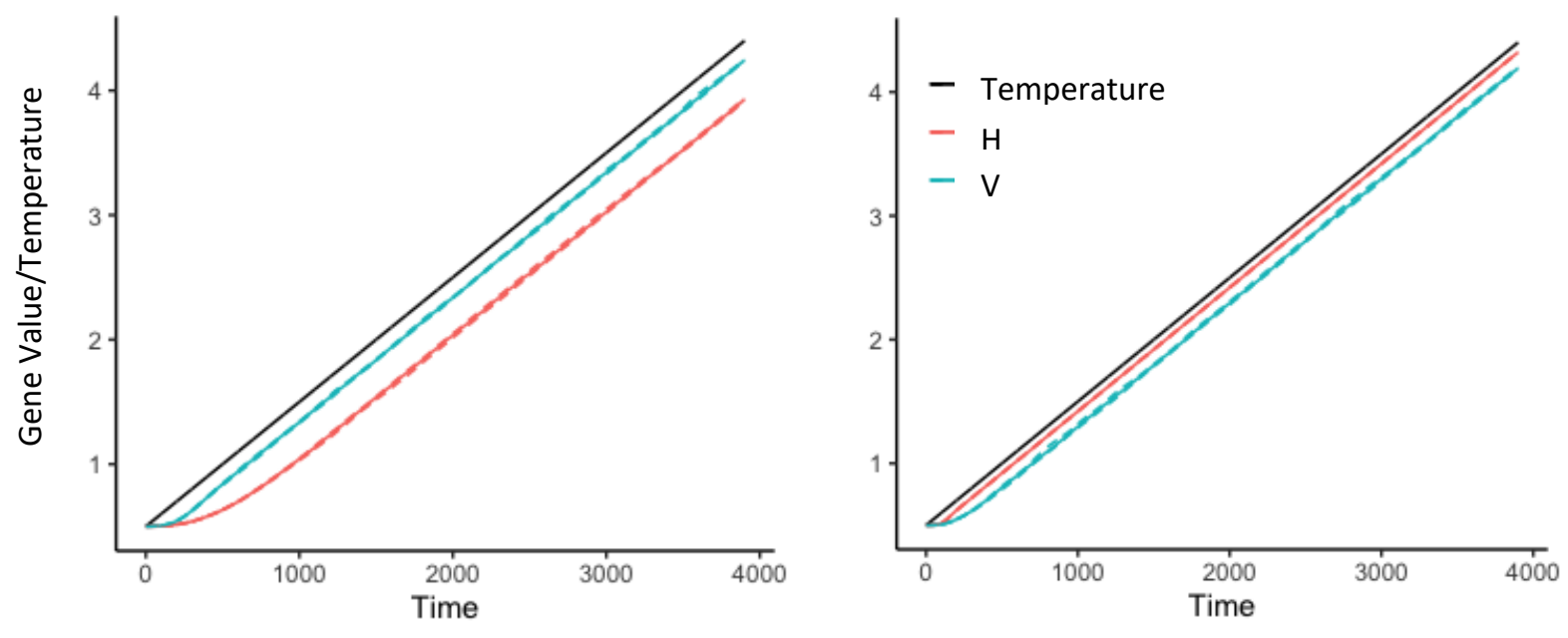

749 Figure 2: Average symbiont gene values (optimal temperature value) of horizontal $(\mathrm{H})$ and

750 vertical (V) transmission mode holobionts in (a) the random transmission model with

751 temperature rate of change per time step $=0.001$ and magnitude of mutation with standard

752 deviation $=0.1$, (b) the optimal transmission model at step $=0.001$, mutation_sd $=0.01$. 

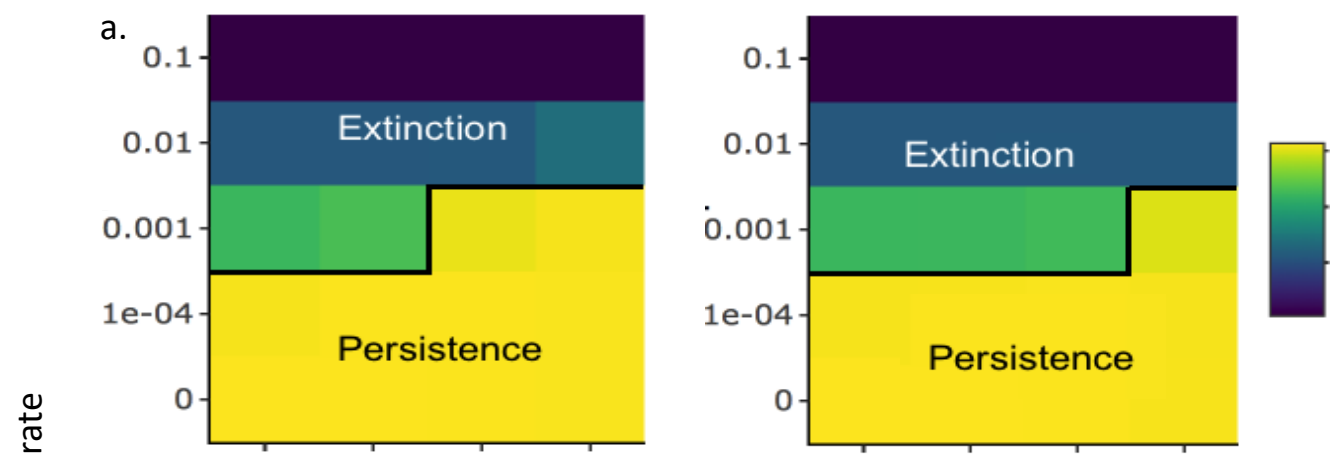

High fitness/ Slow extinction Low fitness/ fast extinction

\section{b.}

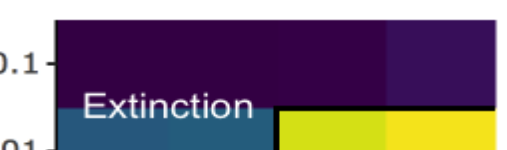

766

767

Figure 3: The dependence of fitness and time to extinction of vertical (V) or horizontal (H)

768 transmission holobionts in separate simulations on rate of temperature change (magnitude

769 of change per time step) and mutation magnitude (standard deviation) with (a) the random

770 transmission model, (b) optimal transmission model. Rapid temperature change (large

771 change per time step) and small magnitude mutations (low mutation variance) are likely to

772 lower holobiont fitness and lead to extinction. The scenarios with persistence are those

773 with high average fitness and those with low fitness have rapid extinction. 

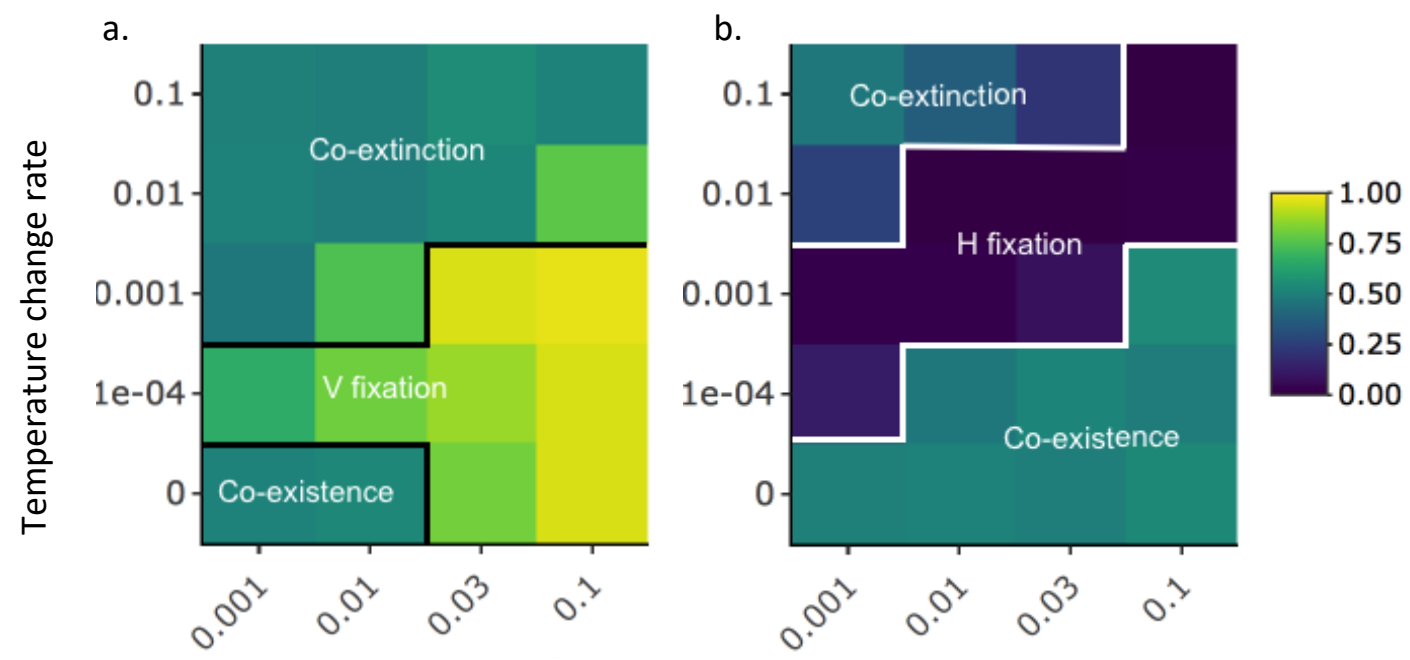

Mutational magnitude (standard deviation)

775 Figure 4. The dependence of average proportion of vertical transmission holobionts in

776 simulations that include both vertical and horizontal transmission types on rate of temperature

777 change (magnitude of change per time step) and mutation magnitude (standard deviation) with

778 (a) random transmission, (b) optimal transmission. Brighter colors indicate conditions in which

779 vertical transmission types reached fixation more quickly (and horizontal types became extinct)

780 and darker colors indicate conditions in which vertical transmission types became extinct faster

781 (and horizontal types reached fixation). 
a.

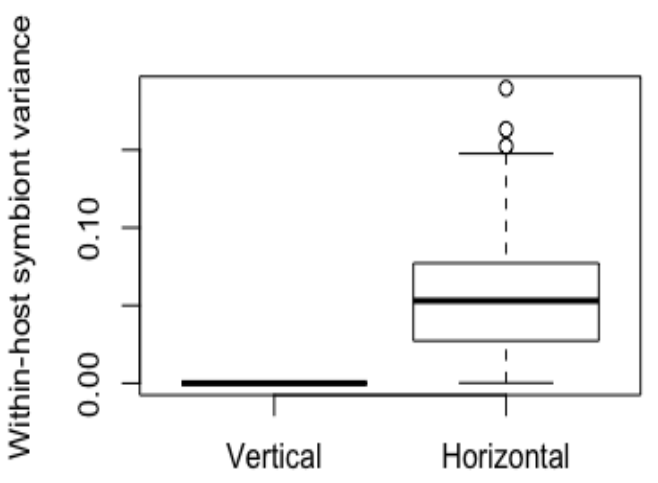

b.

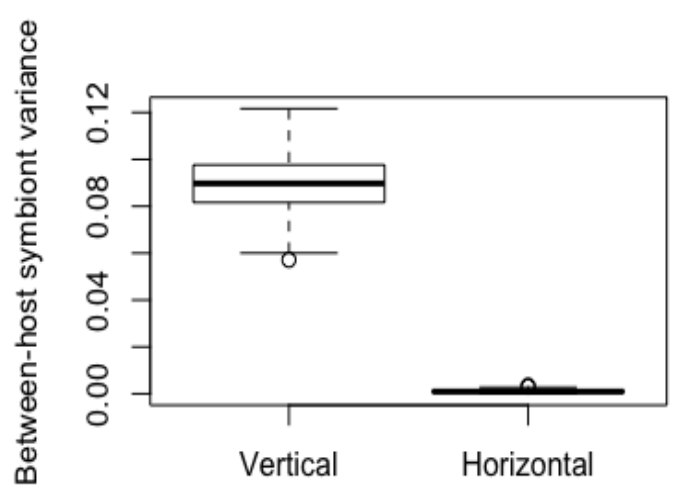

783

784 Figure 5: Variance of symbiont fitness scores (a) within hosts (average of the variance of scores

785 for symbionts within single hosts), (b) between hosts (the variance of the average scores of hosts).

786 Each point is the variance in a single random transmission simulation with only vertical or

787 horizontal transmission types after 1000 time steps. 


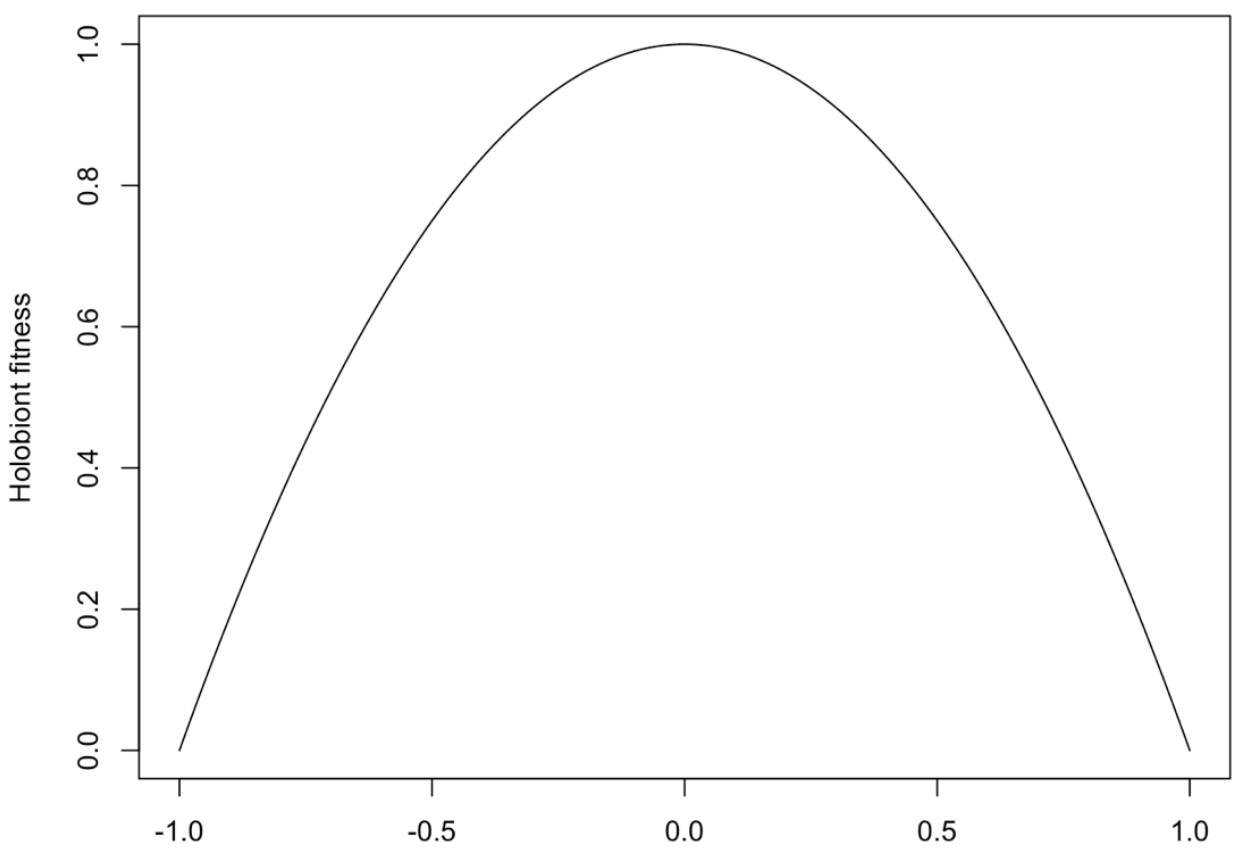

790 Figure 6: Fitness curve. The fitness impact of each symbiont on the holobiont (fitness score) was

791 calculated based the difference between the symbiont gene value (optimal temperature) and the

792 current temperature. When the symbiont gene value matched the current temperature, the fitness

793 score was set equal to 1 and it declined (beta function) as the gene value and temperature were

794 more different. A positive mismatch value indicates the environment is colder than the optimal

795 value for the symbiont and a negative value indicates the environment is colder than the optimal

796 value. 